\title{
Manejo Tecnológico de Lavouras Através de Dispositivos Móveis e Agricultura de Precisão
}

\section{Alternative title: Technological Management of Small Crops Through Mobile Apps and Precision Agriculture}

\author{
Sergio Manuel Serra da Cruz, \\ Diogo Nunes, Carlos Werly, \\ Pedro Vieira Cruz \\ (UFRRJ) \\ serra@ufrrj.br
}

\section{RESUMO}

Cada vez mais os agricultores familiares percebem que a adoção de tecnologia da informação e comunicações sem fio na agricultura é mais que uma tendência, torna-se uma necessidade. Apesar de possuírem acesso à telefonia celular, eles não costumam utilizar aplicativos móveis para auxiliá-los na gestão de suas lavouras. Este trabalho apresenta uma abordagem computacional focada na melhoria da qualidade da cultura do tomate. Apresentamos um ambiente computacional ubíquo e de baixo custo baseado em técnica de agricultura de precisão voltadas para apoiar agricultores na inspeção das lavouras e na deteç̧ão precoce da principal doença que afeta os tomateiros do Brasil. Os resultados iniciais mostraram que a proposta é capaz de manipular observações de campo, descritores de proveniência retrospectiva além de se integrar ao ambiente de processamento baseado em redes neurais das imagens dos tomateiros através das imagens e dados por dispositivos móveis.

\begin{abstract}
Small farmers realize that the adoption of information systems and wireless communications in the field is more than a trend it becomes a necessity. Despite having access to mobile telephony, they do not usually use mobile applications to improve their operations. This work presents a novel computational approach focused on improving the quality of Brazilian tomato crops. We present a low cost and ubiquitous computing environment based on precision agriculture principles to support small farmers to inspect tomato crops and to help them to early detect late blight, one of major disease affecting tomato plants in Brazil. Our initial results have shown that the approach is capable of handling field observations, retrospective provenance descriptors and process images with neural networks to assist small farmers in automated detection of the foliage disease.
\end{abstract}

\section{Categories and Subject Descriptors}

Permission to make digital or hard copies of all or part of this work for personal or classroom use is granted without fee provided that copies are not made or distributed for profit or commercial advantage and that copies bear this notice and the full citation on the first page. To copy otherwise, or republish, to post on servers or to redistribute to lists, requires prior specific permission and/or a fee.

SBSI'15, May 26-29, 2015, Goiânia, Goiás, Brazil.

Copyright 2015 ACM 1-58113-000-0/00/0010 ...\$15.00.

\author{
Marden Manuel Marques \\ (CEASA-RJ)
}

(UFRJ)

\section{marden.marques1@gmail.com}

H [Information Systems]: Models and Principles, Data Model, Information Systems Applications.

\section{General Terms}

Management, Reliability, Experimentation.

\section{Keywords}

Information Management, Provenance, Precision Agriculture, Mobile Devices, Wireless, Late Blight.

\section{INTRODUÇÃO}

O Brasil possui uma agricultura familiar dinâmica e diversificada, composta por 4,3 milhões de pequenos estabelecimentos agrícolas, responsáveis pela produção de produtos da cesta básica. Esta diversidade representa um valor econômico enorme para a agricultura brasileira que experimenta um forte crescimento em sua produtividade [1]. Muitas das recentes descobertas na agricultura de precisão (AP) são resultados do trabalho de equipes multidisciplinares que envolvem agrônomos e cientistas da computação. $\mathrm{Na}$ área agrícola, as tecnologias da informação e comunicação (TICs) abrangem diversos sistemas agrícolas e meios de comunicação, desde os mais antigos como rádios, telefones fixos até mais modernos, como smartphones, computadores, tablets e sistemas multimídia, entre outros. As TICs conferem algum tipo de empoderamento às comunidades de agricultores familiares, profissionalizam as cadeias produtivas; melhoram a gestão e comercialização da produção; tornam os mercados agrícolas mais eficientes e transparentes e melhoram o gerenciamento da terra e dos seus recursos naturais.

Apesar das disparidades regionais entre as zonas urbana e rural, a infraestrutura de telefonia celular vem ampliando sua disseminação no campo com o passar do tempo. De acordo com o Comitê Gestor da Internet [2], a partir do ano de 2010, os lares da zona rural apresentaram o maior crescimento de posse de telefone celular, passando de $58 \%$ em 2009 para $68 \%$ em 2010. Em geral, as comunidades de agricultores familiares possuem acesso à telefonia celular, no entanto não utilizam aplicativos móveis que podem auxiliá-los no alerta ou detecção precoce da ocorrência de doenças nas lavouras ou para auxiliá-los na gestão das lavouras [3]. Essa classe de aplicativos móveis pode ajudar os agricultores a inspecionar a lavoura, reduzir o manejo de agroquímicos nas 
doenças, aumentar seus níveis de renda e oferecer produtos mais saudáveis.

Dentre as culturas anuais com valor expressivo destaca-se o tomate (Lycopersicon esculentum). Ele é a mais importante dentre todas as hortaliças cultivadas no Brasil. O país, oitavo produtor mundial, tem experimentado resultados promissores com os programas de melhoramento genético nos últimos 20 anos, evoluindo de uma produtividade de $37 \mathrm{t} / \mathrm{ha}$, em 1990, para $69 \mathrm{t} / \mathrm{ha}$ em 2011 [1] e [4]. No entanto, a cultura do tomate apresenta grandes desafios. Os tomates são altamente suscetíveis a doenças, pragas e contaminações [5]. O uso indiscriminado de agroquímicos nos tomateiros pode acarretar sérios problemas ao meio ambiente $\mathrm{e}$ à saúde dos produtores e consumidores [6]. Por último, mas não menos importante, os pequenos agricultores nem sempre possuem os recursos necessários para cumprir os padrões cada vez mais rigorosos de segurança alimentar, como a rastreabilidade, verificação de segurança e controle fitossanitário.

Este trabalho tem como objetivo apresentar um ambiente computacional ubíquo que utiliza dispositivos móveis e sistemas Web para auxiliar a inspeção da lavoura e a detecção precoce da requeima, principal doença foliar que afeta os tomateiros brasileiros. $\mathrm{O}$ ambiente se alinha com a temática dos aplicativos móveis na agricultura de precisão [7] e [8], sendo capaz de manipular dados sobre a cultura auxiliando pequenos agricultores a acompanhar seu desenvolvimento e planejar de atividades mais sustentáveis e de maior retorno financeiro. Dentre as tecnologias computacionais adotadas neste trabalho destacamos a engenharia do conhecimento aplicada em sistemas inteligentes em agricultura [9] com ênfase na gestão de dados e descritores de proveniência apoiados pelo metamodelo PROV-DM da W3C [10], além da integração do ambiente com técnicas de inteligência computacional sob a forma de reconhecimento de padrões baseados em redes neuronais tipo Multilayer Perceptron (MLP) [11].

Este trabalho está organizado da seguinte forma, a Seção 2 caracteriza a requeima do tomateiro e os principais conceitos da Agricultura de Precisão, proveniência de dados e correlacionada a abordagem com trabalhos afins. A seção 3 apresenta a metodologia utilizada. A seção 4 apresenta uma proposta de arquitetura distribuída para coletar dados e descritores de proveniência retrospectiva sobre a cultura com vistas a efetuar o reconhecimento dos padrões da doença. As seções 5 e 6 apresentam detalhes da proposta e seus experimentos. A seção 7 conclui o trabalho e aponta limitações e trabalhos futuros.

\section{FUNDAMENTAÇÃO TEÓRICA}

O uso integrado de TICs está se tornando uma realidade em grandes propriedades em diversas partes do mundo [12], contudo seu uso em pequenas propriedades brasileiras é uma novidade bastante desafiadora, ainda mais se considerarmos as disparidades sociais e regionais, as dificuldades fitotécnicas envolvidas na detecção (precoce e automatizada) de doenças e nas resistências de aceitação das novas práticas agrícolas pelos pequenos produtores. Esta seção apresenta os principais conceitos relacionados à área agrícola utilizados neste trabalho.

\subsection{Requeima Em Tomateiros}

O tomateiro ( $L$. esculentum) é proveniente da América do Sul [13]. Sua domesticação foi feita por povos que habitavam a região do México, a partir daí, foi introduzido na Espanha na primeira metade do século XVI, desde então dezenas de cultivares (diferentes tipos de tomates) se espalharam pelo mundo. $\mathrm{O}$ tomateiro é uma planta anual, herbácea, de caule redondo, piloso e macio quando jovem tornando-se fibroso e anguloso com o passar do tempo (Figura 1). As folhas são alternadas, de forma oval a oblonga, compostas de 11 a $32 \mathrm{~cm}$ de comprimento [14] e o fruto climatérico.

Doenças de plantas são anomalias causadas por patógenos (bióticos ou abióticos) que agem continuamente na planta, alterando o seu metabolismo e resultando em queda de produção e/ou perda da qualidade do produto [15]. No tomateiro, as doenças são de frequência/intensidade variadas em função de fatores ou condições (clima, localização da área plantada, modo de implantação e de condução da lavoura) [14]. Já foram relatadas mais de 200 doenças em tomateiros, sendo que a prevalente no Brasil é a requeima. A requeima é a mais destrutiva nas principais regiões produtoras do Brasil. Ela é causada pelo (fungo) Phytophthora infestans. O patógeno ataca a parte aérea das plantas, podendo dizimar uma lavoura em poucos dias, se as condições ambientais forem favoráveis e se medidas de prevenção e controle não forem utilizadas (Figura 1). Os prejuízos causados vão depender do grau de suscetibilidade da cultivar. Estes podem variar de 10 a $100 \%$ da lavoura.

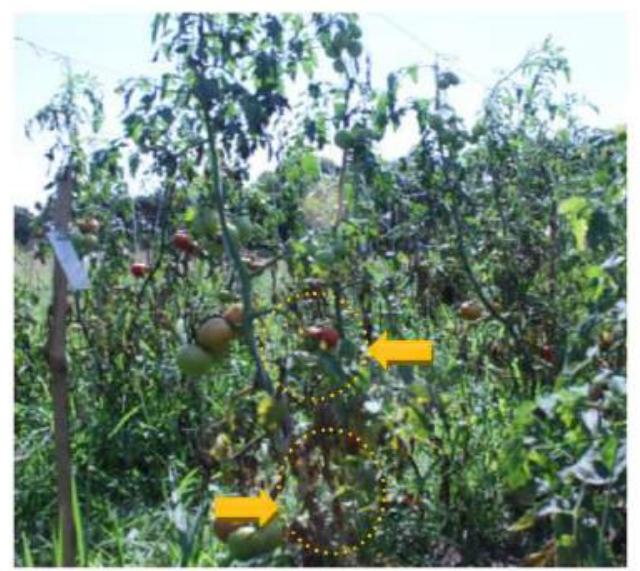

Figura 1. Sintomas de requeima causada por $P$. infestans em tomateiros cultivados no sistema de condução por fitilhos em campo experimental de pesquisas.

A doença é visualmente reconhecida pelo surgimento de pontos escuros nas folhas, cujos matizes variam do cinza ao verdepálido, frequentemente localizadas nas bordas da folha, podendo evoluir para grandes áreas necrosadas marrons [14]. Essas lesões causam a perda de folhas e, nos casos mais severos, a morte da planta. Apesar de tipicamente observados nas folhas, os sintomas também podem aparecer em caules, frutos e brotos [6].

\subsection{Agricultura de Precisão}

Agricultura de Precisão tornou-se um dos principais sistemas de produção agrícola para um uso racional e otimizado dos recursos naturais e insumos agrícolas. [16]. AP é um sistema de manejo integrado que utiliza grandes volumes de dados e hardware e software específicos, baseado nos conceitos de que as variabilidades de tempo e espaço influenciam nos rendimentos das lavouras. AP visa o gerenciamento planejado do sistema de produção agrícola como um todo, não só das aplicações de insumos ou de mapeamentos dos talhões. AP faz uso de um grande 
conjunto de dados e TICs, a saber: GPS, SIG, SI, BD, mecatrônica e sensores para medidas ou detecção de parâmetros ou de alvos de interesse no agroecossistema (água, solo, luminosidade, temperatura, planta, insetos e doenças) [8].

Segundo Coelho e Silva [7], AP não se relacionada apenas com uso de TICs, seus fundamentos podem ser empregados no dia-a-dia das pequenas propriedades pela maior organização, gestão e controle das atividades, dos gastos e produtividade em cada área cultivada. A diferenciação da produção já ocorre na divisão e localização das lavouras dentro da propriedade rural, na divisão dos talhões, ou simplesmente, na identificação de "manchas" que diferem do padrão geral. A partir dessa divisão, o tratamento diferenciado de cada área é a aplicação das técnicas de AP. Figura 2 evidencia os principais conceitos da AP utilizados na modelagem dos aplicativos móveis, Web e do banco de dados deste trabalho.

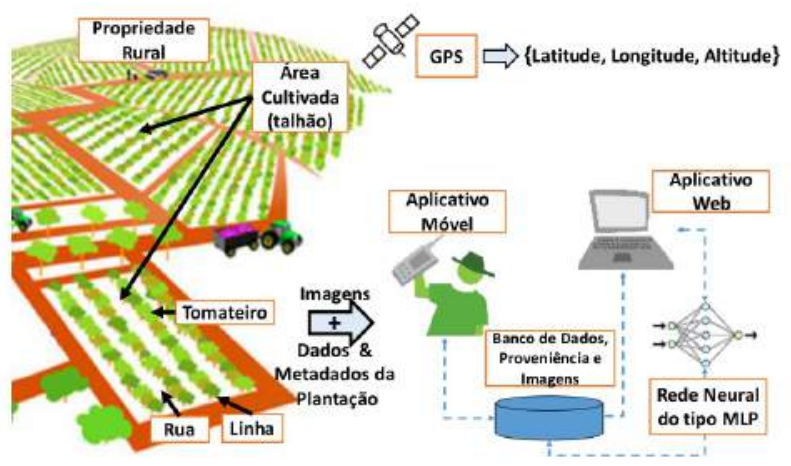

Figura 2. Representação dos principais conceitos da AP utilizados no manejo tecnológico dos tomates.

\subsection{Proveniência de Dados}

Proveniência tem forte presença na área de E-Science, no entanto, sua aplicação vem se difundindo em diversas áreas das TICs [17]. Descritores de proveniência são utilizados em sistemas computacionais para o acompanhamento de histórico, registro, integridade, autenticidade, tolerância a falhas, recuperação de erros, entre outros [18] e [19]. A gestão da proveniência tem por objetivo auxiliar na busca de respostas a inúmeras indagações relacionadas ao experimento científico ou processo computacional. Portanto, para que os dados sejam computados adequadamente $\mathrm{e}$ posteriormente compartilhados (com sucesso), é necessário assegurar que não só sejam livres de falhas e confiáveis, mas também anotados com metadados de proveniência [19].

Segundo Cruz et al [17], Cheney et al [18] e Freire et al. [19], a proveniência pode ser de diversos tipos, podendo ser classificada como prospectiva ou retrospectiva. O primeiro tipo captura a especificação de tarefas computacionais (workflow, programa, atividade, etc.), enquanto o segundo tipo captura as tarefas executadas, dados e parâmetros utilizados, além das informações sobre o ambiente utilizado para derivar um resultado, consistindo em um tipo de histórico estruturado e detalhado sobre a execução de tarefas computacionais. Atualmente, o metamodelo de dados PROV Data Model (PROV-DM) da W3C [10] vem se estabelecendo uma especificação que visa representar e possibilitar a interoperabilidade da proveniência retrospectiva na Web, o metamodelo também vem sendo utilizado em outros tipos de sistemas de informação. PROV-DM possui estruturas que permitem a modelagem dos conceitos essenciais para a representação da proveniência através de três tipos básicos definidos na Tabela 1. Neste trabalho, limitaremos o escopo da captura e representação da proveniência, temos interesse apenas no tipo retrospectiva, ela será coletada na execução do aplicativo móvel e sobre as anotações realizadas pelos agricultores, sendo representado segundo os conceitos essenciais do metamodelo PROV-DM.

Tabela 1. Estrutura essenciais do metamodelo PROV-DM.

\begin{tabular}{|c|l|}
\hline Tipo & \multicolumn{1}{|c|}{ Descrição } \\
\hline $\begin{array}{c}\text { Entidade } \\
\text { (En) }\end{array}$ & $\begin{array}{l}\text { física, digital, conceitual ou outro tipo de coisa com alguns } \\
\text { aspectos fixos; entidades podem ser reais ou imaginárias }\end{array}$ \\
\hline $\begin{array}{c}\text { Atividade } \\
\text { (At) }\end{array}$ & $\begin{array}{l}\text { algum evento que ocorre durante um período de tempo e } \\
\text { age em cima de ou com entidades }\end{array}$ \\
\hline $\begin{array}{c}\text { Agente } \\
\text { (Ag) }\end{array}$ & $\begin{array}{l}\text { relação entre uma entidade e um agente, indicando que a } \\
\text { entidade foi gerada por uma atividade não especificada que } \\
\text { estava associada ao agente }\end{array}$ \\
\hline
\end{tabular}

\subsection{Trabalhos Relacionados}

A oferta de aplicativos móveis na agricultura familiar aumenta a cada dia em todo o mundo, diversos países asiáticos e africanos estão fomentando o desenvolvimento desta classe de aplicativos para empoderar seus agricultores [23]. Por exemplo, nas Filipinas o serviço Farmers Text Center permite que o agricultor transmita imagens (via SMS) para que especialistas avaliem remotamente a ocorrência de doenças nos arrozais. Na Índia, os serviços e-Sagu e $\mathrm{m}$-Krishi provêm orientações técnicas (via SMS) aos agricultores sobre clima, cotações, ocorrência de doenças e formas de manejos de diversas lavouras.

Os agricultores familiares do Brasil dispõem de poucas opções de ferramentas automatizadas que os auxiliem na detecção precoce de doenças. Uma alternativa utilizada é a chave de classificação manual definida por Correa et al [6]. Essa chave está baseada em imagens estilizadas de folhas de tomates que quantificam o grau de infestação por $P$. infestans, permitido avaliar visualmente o grau de contaminação do tomateiro. A detecção manual precoce de doenças, realizada pelos especialistas, nem sempre é uma opção economicamente viável. No entanto, do ponto de vista da detecção automatizada de doenças foliares em diversas plantas, já existem diversos trabalhos relacionados.

Sanyal e Patel [20] conduziram um estudo do tipo simulação de análise de textura e cor das folhas de arroz (Oryza sp.) para identificação das doenças brown spots e blast diseases. A simulação realizada baseou-se em 400 amostras e a classificação foi realizada por uma rede MLP. Foram utilizadas imagens de folhas doentes e normais e chegou-se a um desempenho de $89,26 \%$ de acertos na classificação individual dos pixels das imagens. Camargo [21] define um classificador baseado em uma máquina de vetor de suporte (SVM) para operar sobre as imagens coloridas de algodão (Gossypium sp.). Foram utilizadas 127 imagens de culturas de algodão. $O$ melhor modelo de classificação foi encontrado com 45 características, chegando a $93,1 \%$ de desempenho.

Vieira et al. [22] utilizou uma rede neural MLP para detectar as lesões ocasionadas em folhas de tomate. No entanto, suas taxas de acertos foram de apenas $77,8 \%$ na identificação de lesões por requeima. Além disso, dentre os testes conduzidos, foram utilizadas poucas amostras, apenas 9 amostras de folhas com lesões da doença de um conjunto total de 65 amostras de folhas lesionadas por três tipos diferentes de doenças. Neste estudo, existe um fator limitante quando comparado a nossa abordagem, isto é, as 
imagens digitais sofreram pré-processamento manual, onde o usuário delimita as manchas mais relevantes da imagem original. Além disso, as imagens foram coletadas em ambiente de laboratório e não há informações sobre o cultivar investigado.

Por fim, vale ressaltar que apesar dos nossos esforços durante a fase de levantamento quase-sistemático de dados na literatura, não fomos capazes de localizar trabalhos relacionados que utilizem aplicativos móveis, técnicas e agricultura de precisão e descritores de proveniência retrospectiva para processamentos de dados apoiados em redes neurais que auxiliam a detecção precoce de requeima em tomateiros.

\section{METODOLOGIA}

Neste trabalho, além do levantamento de dados na literatura, utilizamos duas metodologias destinadas ao desenvolvimento da arquitetura. A primeira foi aplicada na concepção e projeto dos aplicativos móvel e Web, permitindo: compreender as necessidades e desejos dos pequenos agricultores; conceber soluções ubíquas, multidisciplinares, inovadoras e inclusivas que satisfazem as necessidades dos agricultores e, finalmente oferecer uma solução computacional que é acessível, fácil de ser utilizada, sustentável, barata e computacionalmente viável. A segunda é utilizada na modelagem conceitual dos aplicativos e dos bancos de dados, ele permitiu documentar o processo de elaboração da solução computacional.

\subsection{Metodologia Centrada no Agricultor}

Esta metodologia está baseada no paradigma HumanCentered Design (HCD) [24] e [25], inicialmente apresentado por Krippendorff [26] e tem o indivíduo como elemento central no projeto e desenvolvimento de produtos e serviços. A metodologia IDEO HCD se apresenta como um toolikt que possui três fases: Ouvir, Criar e Implementar (Figura 3). Durante o processo, o pensamento o analista alternará do nível concreto ao abstrato, identificando temas e oportunidades e, mais tarde, retornará ao concreto com soluções e protótipos.

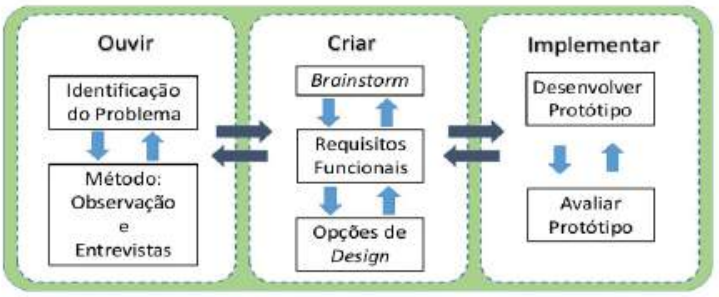

Figura 3. Representação Conceitual da IDEO HCD.

As fases três fases do IDEO HCD são definidas da Tabela 2.

Como primeira fase do processo, ocorrem consultas os usuários (agricultores familiares, técnicos agrícolas, agrônomos e pesquisadores) para identificar os problemas e dificuldades inerentes à cultura do tomate. Durante esta fase, dedica-se a maior parte do tempo a ouvir o que os usuários tinham a dizer sobre os desafios que enfrentam em suas lavouras. Isto proporciona o meio para captar relevantes informações para a identificação dos problemas do monitoramento dos tomateiros e gestão de dados agrícolas. A fim de obter essas informações se realizaram entrevistas abertas e visitas de observações no campo experimental.
A segunda fase utiliza informações obtidas na fase anterior para identificar os requisitos funcionais do sistema proposto. Durante o processo de criação, se desenvolvem sessões de brainstorming com os usuários. Durante as sessões os usuários discutem abertamente o que eles acham que seria um projeto interessante e o que sistema proposto deveria fazer a fim de auxiliar o monitoramento e gerencia dos tomateiros. Adotamos esboços, cenários e protótipos de papel, a fim de ilustrar e visualizar ideias. Os resultados das sessões auxiliam na definição dos requisitos funcionais do sistema móvel e nas opções de projeto do aplicativo móvel.

Tabela 2. Descrição das fases do IDEO HCD

\begin{tabular}{|c|l|}
\hline Fase & \multicolumn{1}{c|}{ Descrição } \\
\hline Ouvir & $\begin{array}{l}\text { O ato de projetar soluções inovadoras e relevantes, que } \\
\text { atendem às necessidades dos usuários, começa com a } \\
\text { identificação de suas necessidades, expectativas e aspirações } \\
\text { para o futuro. Essa fase diz respeito a como abordar as } \\
\text { pessoas em seus próprios contextos para entender em } \\
\text { profundidade os seus problemas. }\end{array}$ \\
\hline Criar & $\begin{array}{l}\text { Para transformar pesquisas em soluções para o mundo real, é } \\
\text { preciso passar por um processo intermediário de síntese e } \\
\text { interpretação. Isso requer filtrar e selecionar a informação, } \\
\text { traduzindo insights sobre a realidade atual em oportunidades } \\
\text { para o futuro. Esta é a parte mais abstrata do processo, } \\
\text { quando se deve transformar necessidades concretas dos } \\
\text { usuários em insights mais gerais. Com as oportunidades } \\
\text { definidas, deverá ser adotado um ponto de vista genérico } \\
\text { para criar diversas soluções em brainstorms e elicitar os } \\
\text { requisitos e converter algumas delas em protótipos. }\end{array}$ \\
\hline Imple- & $\begin{array}{l}\text { Essa fase desafia a equipe de desenvolvimento a desenvolver } \\
\text { os elementos necessários para que a solução tenha sucesso e } \\
\text { para monitorar o seu impacto. }\end{array}$ \\
\hline mentar
\end{tabular}

A terceira fase do processo, utilizamos os resultados das duas primeiras fases o para desenvolver rápida prototipação do aplicativo móvel proposto.

\subsection{Metodologias Centradas na Modelagem dos Artefatos Computacionais}

Ainda no decorrer da segunda fase do IDEO HCD começamos a desenvolver os artefatos computacionais tradicionais (e.g. projetos, telas, modelos, diagramas) destinados ao consumo por parte de profissionais de computação. Por esse motivo, foi necessário utilizar um segundo conjunto de metodologias específicas para a área de Sistemas de Informação que permitiram integrar os desenvolvimentos dos aplicativos móveis e Web. Por exemplo, adotamos técnicas de modelagem UML com design patterns para o desenvolvimento do aplicativo móveis, a modelagem relacional de dados juntamente com a especificação PROV-DM para a modelagem conceitual do banco de dados e de descritores de proveniência [17] e [19].

\section{ARQUITETURA PROPOSTA}

Existem vários referenciais teóricos para o desenvolvimento de aplicações móveis. Nossa arquitetura adotou o modelo de múltiplas camadas com módulos de baixo acoplamento apresentado por Abrahamsson [27]. A arquitetura é composta por camadas (Figura 4): aplicativos Móvel, Web, além dos módulos de redes neurais e dos repositórios de dados e metadados de proveniência. As principais funcionalidades do ambiente foram elicitadas com técnicas de análise dos requisitos levantados junto aos agricultores, agrônomos e técnicos. 
O aplicativo móvel foi concebido para ter interfaces gráficas (bem simples) para serem utilizadas diretamente na lavoura pelos agricultores que possuam pouca experiência em TICs. Através dele, o agricultor será capaz de registrar as informações sobre as áreas cultivadas (ruas e linhas), coletar dados de inspeção e imagens sobre os tomateiros da sua lavoura e fazer anotações.

O banco de dados da arquitetura é do tipo distribuído, foi concebido para armazenar os dados (permanentes e temporários) sobre as lavouras, áreas cultivadas, talhões, plantas, imagens dos espécimes e cultivares, dados de GPS, procedimentos agrícolas, dados ambientais e também dados de proveniência retrospectiva sobre o monitoramento das lavouras realizado pelos agricultores através dos dispositivos móveis. O banco de dados também é capaz de armazenar, no desktop, os dados históricos das sucessivas coletas de informações sobre as lavouras, além de descritores de proveniência do tipo retrospectiva, dados e imagens utilizados pela rede neural.
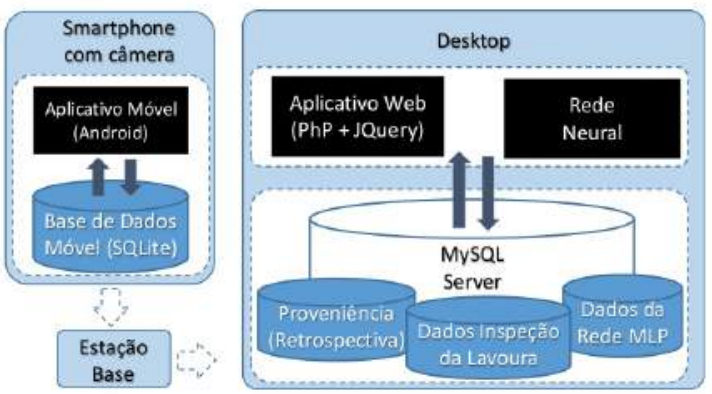

Figura 4. Representação conceitual da arquitetura.

Os dados de inspeção e descritores de proveniência são transferidos do dispositivo móvel para o desktop através de uma estação base que executa o aplicativo Web e a rede MLP (à psoteriori). O aplicativo Web é capaz de manter informações sobre as propriedades rurais, áreas de cultivo, lavouras, mão de obra, aplicações de defensivos, anotações de cultivo, estados e eventos meteorológicos, (re)ocorrência de doenças e pragas. Ele também é capaz de importar os dados da aplicação móvel e produzir os relatórios gerenciais consolidados de acompanhamento a serem submetidos aos serviços de assistência agronômica. O aplicativo Web também desempenha um papel importante pois é através dessa interface que se executam as tarefas de reconhecimento de padrões para a detecção automatizada de requeima baseadas em redes neuronais do tipo MLP.

Adotamos o pattern MVC (Model-View-Controller) na arquitetura proposta para promover a interligação entre os aplicativos, pois ele oferece flexibilidade e nítida separação de responsabilidades. Isto é, ao se separar o controlador do modelo da apresentação, o fluxo da aplicação poder ser facilmente isolado e ao se separar o modelo da apresentação, o modelo é isolado de alterações. Adicionalmente, é possível utilizar a rede de dados de modo intermitente e ainda permanecer útil quando desconectado no campo, a porção cliente da aplicação decide sobre quando buscar os dados do servidor e quando buscar os dados do armazenamento local. As estratégias de distribuição de dados locais podem ser baseadas em cache ou sincronização para melhorar a responsividade e manter a coerência dos dados.

\section{ARTEFATOS COMPUTACIONAIS}

Esta seção apresenta as principais características dos artefatos que fazem parte do ambiente de detecção precoce de requeima em tomateiros.

\subsection{Aplicativo Móvel}

O aplicativo móvel foi desenvolvido em Java e PHP (Figura 5), sendo baseado no sistema Android (versão 5.0.1), opera em simples smartphones e não requer que a área cultivada seja atendida pela rede de telefonia celular.

O aplicativo é operado pelo agricultor que é capaz de coletar dados e armazenar imagens georreferenciadas sobre os tomateiros infectados (ou suspeitos) através da câmera embutida. Além disso, permite que o agricultor registre e identifique unicamente cada tomateiro da propriedade através do retículo Rua-Linha-Talhão. Cada tomateiro possui uma identificação única, descritores e imagens que representam seu estado geral que podem variar ao longo do tempo desde o plantio até a colheita dos frutos do tomate.
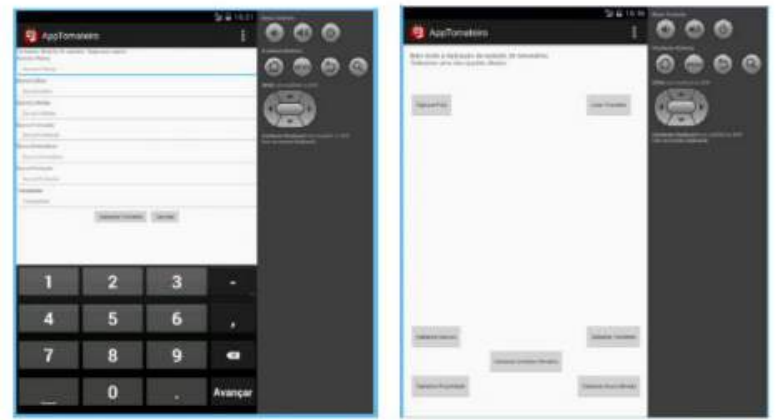

Figura 5. Exemplos de telas do aplicativo móvel.

Os dados de inspeção da lavoura e descritores de proveniência coletados pelo aplicativo móvel são armazenados local e temporariamente na biblioteca SQLite que implementa um banco de dados transacional compatível com padrão SQL de modo embutido no dispositivo móvel. Posteriormente, os dados são transferidos para o SGBD MySQL. O banco de dados foi idealizado para armazenar informações textuais, imagens e descritores de proveniência retrospectiva (Figura 7). O modelo conceitual foi implementado para representar os atributos que são utilizados pelos aplicativos móvel e Web e pela rede neural MLP.

\subsection{Conectividades Entre Aplicativos}

A Figura 6 representa a responsividade entre os artefatos, os clientes são os aplicativos móvel e web utilizados pelo agricultor para se conectar ao desktop (que atua como o servidor). A motivação para utilizar as tecnologias PHP/MySQL foi a facilidade de interação com os bancos de dados e clareza de comunicação com as estruturas empregadas nesta pesquisa.

A conectividade entre os aplicativos está centrada nos protocolos HTTP e HTTPS, onde o Android realiza conexão com o PHP e implementa o sistema de registro integrando o PHP, MySQL e SQLite através de scripts JSON [28] que são tecnologicamente neutros, abertos e independente de linguagem, facilitando a troca de dados entre as plataformas. A sincronização de dados entre o SQLite (base de dados móveis) e o servidor MySQL (repositórios de dados e de proveniência) é realizada por esses scripts. Um script JSON é dinamicamente construído para 
representar os dados/imagens/proveniência, lançando-os para classe AsyncHttpClient do PHP no servidor que o decodifica e insere os dados no banco relacional.

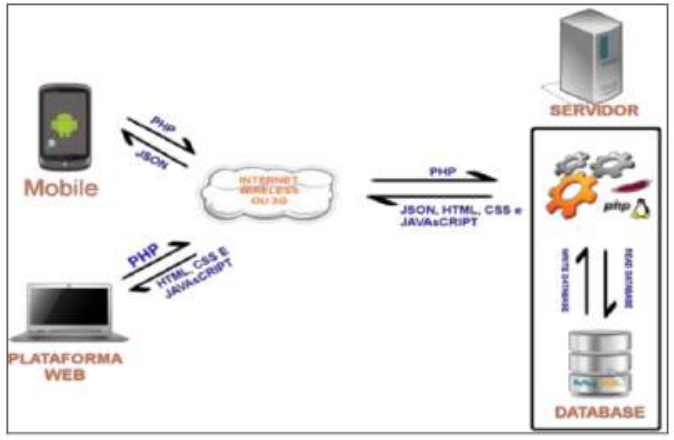

Figura 6. Representação esquemática da conectividade entre os clientes e o servidor.

\subsection{Aplicativo Web}

$\mathrm{O}$ aplicativo Web utiliza recursos tradicionais da Web (PHP, HTML, JQuery, CSS e MySQL), visando tornar a aplicação leve, de manutenção simplificada e capaz de ser executada em desktops não muito potentes. $\mathrm{O}$ aplicativo possui interfaces simplificadas, perfis distintos e pode ser operado pelo próprio agricultor ou por técnicos agrícolas e agrônomos (previamente cadastrados). O aplicativo Web carrega automaticamente os dados de inspeção e descritores de proveniência obtidos através do aplicativo móvel sempre que este se conectar à Internet. Graças aos scripts JSON, o aplicativo móvel faz o upload de dados e o aplicativo Web efetua verificações de dados, garantindo integridade e evitando a duplicação dos dados (previamente carregados).

Além disso, o aplicativo Web possui rotinas gerenciais para emissão de históricos e relatórios parametrizáveis (com diferentes graus de consolidação) que mapeiam as lavouras e seus manipuladores, colheitas realizadas em diferentes períodos e (re)utilização das áreas cultivadas (talhões), permitindo avaliar quais regiões (ruas e linhas) houve maior incidência de doenças, quais os tomateiros são mais afetados, quais cultivares mais resistentes, também é possível consultar as anotações sobre condições ambientais nas lavouras, entre outros. Por fim, o aplicativo Web é capaz de invocar o módulo de redes neurais para realizar o reconhecimento de padrões da requeima, notificando ao agricultor caso algum tomateiro possua indícios da doença, permitindo que ele tome medidas adequadas precocemente.

\subsection{Bancos de Dados e de Proveniência}

A Figura 7 ilustra apenas as principais classes do modelo conceitual do banco de dados necessárias à compreensão deste trabalho. Representamos apenas as mais importantes sobre os dados históricos da lavoura e os principais descritores de proveniência do tipo retrospectiva. Observe que no texto (ao lado do nome das classes) existe uma indicação de correlação da classe segundo estrutura básica do metamodelo PROV-DM ao qual estão classificados. Esta correção está apoiada nas classificações apresentadas por Cruz et al [17].

A classe Propriedade Rural (En) é uma localidade na microregião estudada, contém descritores sobre fatores intrínsecos da mesma, como qualidade da água, topografia predominante, mecanização e seu tipo. Já a classe Área Cultivada (En) armazena dados sobre o cultivo realizado, assim como, a estrutura da área e a qualidade do solo pertencente ao local. A classe Rua (En) trata do dimensionamento e espaço físico que o produtor rural tem para se locomover entre as linhas e para manipular os tomateiros, cada rua possui duas Linhas (En) de tomateiros, sendo que este relacionamento terá a quantidade de linhas e a distância entre as mesmas.

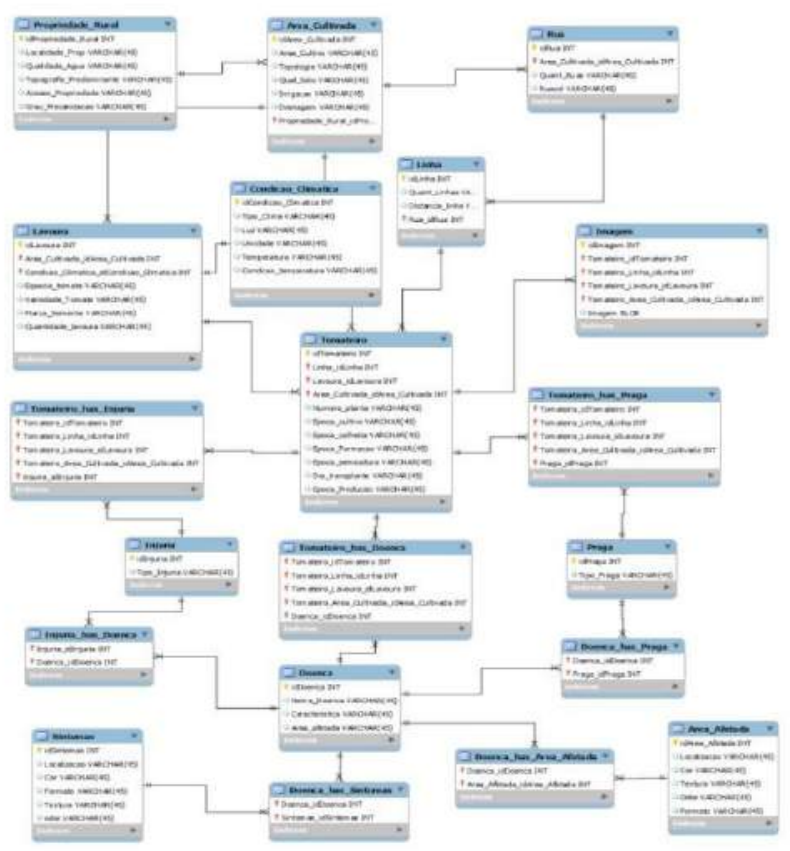

Figura 7. Fragmento do DER utilizado pela arquitetura.

A classe Condição Climática (At) armazena as características climáticas predominante da propriedade (luminosidade, temperatura, umidade) influenciam significativamente na cultura do tomate. A classe Lavoura (At) descreve a técnicas de cultivo e os genótipos (cultivares) de tomate, dados da semente, assim como a quantidade de plantas da lavoura, entre outros. As classes Praga, Doença e Injuria (At) contém informações referentes ao controle de insetos e ácaros (pragas) e doenças dos tomateiros. Injuria são pequenas lesões ocasionadas na planta que cria condições propícias ao surgimento dos itens citados anteriormente.

A classe Tomateiro (En) armazena os dados específicos do ciclo de vida dos tomateiros (épocas de cultivo, colheita, formação, semeadura, produção e transplante). Todos estes itens citados juntamente com a entidade Imagem são utilizados pelas redes neurais. Cada imagem de tomateiro pode ser georeferenciada permitindo detectar automaticamente a requeima e também avaliar o deslocamento da doença pelos talhões e pela propriedade. As classes Proprietário e Agricultor (Ag), não representadas na Figura 7, armazena os dados do proprietário da propriedade agrícola ou área cultivada e sobre o trabalhador que atua na área ou que coleta os dados sobre as lavouras.

\subsection{Rede Neural MLP}

A incorporação de técnicas inteligência computacional na arquitetura tem como objetivo automatizar o processo de identificação da requeima. Neste trabalho, por limitações de 
escopo, não discutiremos as especificidades das redes neurais. A tecnologia computacional adotada foi baseada na inteligência computacional, sob a forma de reconhecimento de padrões baseados em redes neurais do tipo MLP.

\section{AVALIAÇÃO EXPERIMENTAL}

O conjunto de amostras digitais utilizados neste experimento perfaz um total de 226 imagens digitais dos tomateiros obtidas de amostras de 66 cultivares plantados em campos experimentais (talhões) do setor de horticultura do Departamento de Fitotecnia do IA/UFRRJ, uma área historicamente associada com a ocorrência natural da requeima. Os genótipos incluem um cultivar comercial suscetível à doença, dois resistentes e 63 ainda sob avaliação na Universidade. Como instrumentos de experimentação utilizamos um smartphone (processador $1.2 \mathrm{GHZ}, 8 \mathrm{MB}$ de RAM, câmera de 5 MP e GPS) para executar o aplicativo móvel e um desktop com processador Intel Core $\mathrm{I} 3 \mathrm{com} 4 \mathrm{~GB}$ de RAM e $500 \mathrm{MB}$ de disco rígido para executar o aplicativo Web.

Resumidamente, os dados de inspeção, descritores de proveniência e imagens dos tomateiros foram coletados durante visitas a um talhão ( 0,3 hectare) do campo experimental do IA/UFRRJ. Os dados foram coletados por técnico agrícola ao percorrer suas ruas e linhas. A distribuição da amostragem das imagens deu-se pelo princípio da casualização. Os dados, descritores e imagens foram transferidos entre os aplicativos para serem posteriormente processados pela rede neural do tipo MLP.

O processamento da rede neural foi realizado em três etapas sobre cada amostra colorida de tomateiro: (a) a aplicação de um filtro vermelho/verde e redução da qualidade das imagens para 96 dpi; (b) conversão para imagem em tons de cinza e (c) análise de relevância de dados das imagens. Sobre cada imagem digital, foram realizadas contagens de pixels que se encontram dentro de faixas de cores ou tons de cinza relevantes. A identificação dos tomateiros e áreas foliares atingidas pelas requeima utiliza as imagens coletadas pelo aplicativo móvel diretamente no talhão sob iluminação solar direta sem aplicação de tratamentos prévios ou efeitos de qualquer natureza sobre as imagens.

O processamento da imagem do tipo filtragem vermelho/verde é um procedimento automatizado que considera o princípio de que a cor da planta saudável é a verde intenso, a redução da qualidade visa acelerar o processamento das rotinas de contagem, reduzir o volume de armazenamento de dados no banco de dados. Em seguida, é realizada a análise do tom de cada pixel da imagem, classificando-o dentro de uma das seguintes opções:

(i) um pixel é classificado como doente e será convertido para a cor vermelha $(255,0,0)$, no sistema RGB, caso seja de um tom amarelado ou marrom, indicativos de algum tipo de lesão.

(ii) um pixel é classificado como sadio e será convertido para a cor verde $(0,255,0)$ caso seja de um tom esverdeado, podendo ir de uma matiz mais clara até um verde mais escuro;

(iii) um pixel de qualquer tom diferente dos anteriores será considerado como fundo da imagem e será convertido para preto.

As primeiras avaliações experimentais da arquitetura realizadas com as imagens coletadas através do aplicativo móvel utilizaram contagens de pixels realizadas sobre as imagens. Apenas aquelas que possuíam descritores de proveniência que indicavam a possível presença de requeima foram utilizadas na rede neural.

Em função dessa análise, foram pré-selecionadas para o treinamento das redes neurais oito variáveis, onde cada uma contém informações sobre quantidades de pixels em uma determinada faixa de cor ou tom de cinza. A classificação final das amostras processadas por uma rede neural MLP, com configurações variadas, alcançaram um desempenho médio aproximado de $88 \%$ de acertos, valores compatíveis com a literatura (a descrição detalhada das redes neurais está presente em nossos trabalhos anteriores) [29] e [30].

\section{CONCLUSÃO}

Se por um lado os mercados consumidores estão cada vez mais exigentes com relação à rastreabilidade e respeito ao meio ambiente. Por outro lado, os produtores percebem que existem poucos sistemas computacionais que os auxiliem na tomada de decisôes que afetam diretamente suas lavouras. O uso intensivo de técnicas de AP, TICs e sistemas inteligentes aplicados à agricultura são mais que uma tendência, tornam-se uma questão de necessidade.

Este trabalho apresentou uma abordagem computacional ubíqua que apresenta os primeiros resultados de ordem prática. Os artefatos computacionais de baixo acoplamento e dispostos em múltiplas camadas processam dados e descritores de proveniência que poderão ser utilizados na agricultura familiar para auxiliar na detecção precoce da requeima em tomateiros e possivelmente aumentar a produtividade da lavoura de tomate e gerar produtos de maior valor agregado, mitigando os problemas decorrentes da doença. A principal vantagem da abordagem, além da sua mobilidade, é oferecer oportunidades para os pequenos agricultores para executar suas operações de forma mais produtiva.

Os artefatos foram concebidos após interações e participações centradas nas necessidades dos agricultores familiares graças à adoção da metodologia IDEO-HCD. Os artefatos foram idealizados para acolher necessidades ainda não atendidas apontadas pelos usuários, explorando a ubiquidade e a mobilidade oferecidas pelo uso da comunicação sem fio no campo. $\mathrm{O}$ trabalho utilizou apenas equipamentos (smartphones e desktops) de baixo custo, software livres e desenvolveu interfaces que visam a facilidade de uso pois os agricultores têm pouco espaço para erros e pouca (ou nenhuma) experiência em processamento digital com programas sofisticados ou mesmo o uso de técnicas de redes neurais e bancos de dados.

Investigaçôes e experimentações adicionais ainda se fazem necessários, em especial no que diz respeito aos estudos agronômicos mais aprofundados e aos estudos quantitativos das definições das divisões dos talhões, princípios de amostragem e casualização das amostras, frequências de inspeção, entre outros.

Como trabalhos futuros serão avaliadas melhorias na integração entre os artefatos com os algoritmos de processamento digital de imagens, uma vez que a qualidade dos dados dos descritores de proveniência de entrada é um fator de impacto para o desempenho de um sistema de reconhecimento baseado em redes neurais e análise de dados. Um outro conjunto de atividades diz respeito à avaliação dos pesquisadores IA/UFRRJ com vista a qualificar, segundo a ótica fitossanitária, a corretude da solução computacional comparada ao método desenvolvido por [6]. 


\section{AGRADECIMENTOS}

Agradecemos à FAPERJ (processos E-26/112.588/2012 e E26/111.494/2012) e ao CYTED2014 (P514RT0013) pelos financiamentos, ao PIBIC/CNPq, ao PIBITI/Funttel e ao MEC/SESu pelas bolsas. Agradecimentos ao IA/UFRRJ, aos professores Antônio Abboud e Margarida G. F. do Carmo e ao Laboratório de Bancos de Dados da UFRRJ.

\section{REFERÊNCIAS}

[1] IBGE. 2013. Brasil em números". hHttp://biblioteca.ibge.gov.br/visualizacao/ periodicos/2/bn_2013_v21.pdf

[2] CGI - Comitê Gestor Da Internet No Brasil. 2011. TICs domicílios e empresas 2010". São Paulo, 2011. http://www.cetic.br/tic/2010/index.htm

[3] Schwartz, C. 2012. Relações de Gênero e Apropriação de Tecnologias de Informação e Comunicação na Agricultura Familiar de Santa Maria-RS". Tese de Doutorado - UFSM.

[4] FAOSTAT. 2015. http://faostat.fao.org/site/291/default.aspx

[5] Nakano, O. 1999. As pragas das hortaliças: seu controle e o selo verde". Horticultura Brasileira, Brasília, DF, v. 17, n. 1, p. 4-5.

[6] Correa, F.M., Bueno Filho, J.S.S., and Carmo, M.G.F., 2009. Comparison of Three Diagrammatic Keys for the Quantification of Late Blight in Tomato Leaves, Plant Pathology 58, p.1128-1133.

[7] Coelho, J. P. C. and Silva, J. R. M. 2011. Agricultura de Precisão.

http://agrinov.ajap.pt/manuais/Manual_Agricultura_de_Precis ao.pdf.

[8] MAPA. 2014. Ministério da Agricultura, Pecuária e Abastecimento. Agricultura de precisão Secretaria de Desenvolvimento Agropecuário e Cooperativismo. Brasília. http://www.agricultura.gov.br/arq_editor/Boletimtecnico.pdf.

[9] Quincozes, E. R. F. et al. 2010. Gestão Do Conhecimento Aplicada A Uma Organização Intensiva Em Conhecimento: O Caso Da Embrapa Clima Temperado", Interscience Place, n. 3 p.123-141.

[10] Moreau, L. and Missier, P. 2013. PROV-DM: The PROV Data Model", W3C, www.w3.org/TR/prov-dm/.

[11] Johnson, R. and Wichern, D. 1992. Applied Multivariate Statistical Analysis. Prentice-Hall International, Inc. p.356394, p.52-85.

[12] Wang, N., et al. 2006. Wireless sensors in agriculture and food industry-Recente development and Future perpective. Computer and Eletronics in Agriculture, n.50, p.1-14.

[13] Bergougnoux, V. 2014. The history of tomato: from domestication to biopharming. Biotechnol Adv. 2014 JanFeb, 32(1):170-89.

[14] Filgueira, F. A. R. 2008. O novo manual de olericultura, Editora da UFV. 3a edição.

[15] DISQUAL. 2010. Otimização da qualidade e redução de custos na cadeia de distribuição de produtos hortifrutícolas frescos - Manual de Boas Práticas. http://www2.esb.ucp.pt/twt/disqual/pdfs/disqual_tomate.pdf.

[16] Adrian A.M., Norwood, S.H., Mask, P.L. 2005. Producers perceptions and attitudes toward precision agriculture technologies. Comput. Electronics Agric. 48(3): 256-271.

[17] Cruz, S. M. S., Campos, M., Mattoso, M., Towards a Taxonomy of Provenance in Scientific Workflow Management Systems. In IEEE Int Workshop on Scientific Workflows, 2009, Los Angeles, USA.

[18] Cheney, J., Chiticariu, L., Tan, W.C. 2009. Provenance in databases: Why, how, and where. Foundations and Trends in Databases 1 (4), 379-474.

[19] Freire, J., Koop, D., Santos, E., Silva, C.T. 2008. Provenance for computational tasks: A survey. Computing in Science \& Engineering $10(3), 11-21$.

[20] Sanyal, P. and Patel, S. C. 2008. Pattern recognition method to detect two diseases in rice plants Imaging Science Journal, v.56, n.6, p. 319-325.

[21] Camargo, A. and Smith, J.S. 2009. Image Pattern Classification for the Identification of Disease Causing Agents in Plants", Computers and Electronics in Agriculture 66, p.121-125.

[22] Vieira, F. S. et. al., 2011. Utilização de Processamento Digital de Imagens e Redes Neurais Artificiais para Diagnosticar Doenças Fúngicas na Cultura do Tomate. Anais do XX Seminário de Computação, p. 58-69.

[23] Brugger F. 2011. Mobile applications in agriculture. Syngenta Foundation.

[24] IDEO. HCD - Human Centered Design: Kit de ferramentas.: Ideo, 2009. 102p. http://www.ideo.com/work/humancentered-design-toolkit/.

[25] Brown, T. 2010. Design Thinking: Uma Metodologia poderosa para Decretar o Fim das Velhas Ideias. Ed Campus. 1a edição Abrahamsson, P. 2005. Mobile software development - the business opportunity of today. Proc. of the International Conference on Software Development, p. 20-23.

[26] Krippendorff, K. 2000. Propositions of Human-centeredness: A Philosophy for Design. In: DURLING, D. FRIEDMAN, K. (Eds.). Doctoral Education in Design: Foundations for the Future. Staffordshire (UK): Staffordshire Univ.Press. p.55-63.

[27] Abrahamsson, P. 2005. Mobile software development-the business opportunity of today. Proceedings of the International Conference on Software Development, p.20-23.

[28] JSON - JavaScript Object Notation - Disponível em http://www.json.org/json-pt.html

[29] Vianna, G.K. e Cruz, S.M.S. (2013) “Análise Inteligente de Imagens Digitais no Monitoramento da Requeima dos Tomateiros". Anais do IX Congresso Brasileiro de Agroinformática, Brasil.

[30] Vianna, G.K. e Cruz, S.M.S. (2014) "Using Multilayer Perceptron Networks in Early Detection of Late Blight Disease in Tomato Leaves". Proc. of the 16th ICAI, USA. 